\title{
Substituent Effect on AIE Mechanism of Two Coumarin Derivatives: Uncommon TICT Fluorescence in Aggregation State
}

\section{Qiao Li}

Hainan Normal University

\section{Yang Zhao}

Hainan Normal University

Zhigang Niu

Hainan Normal University

Enju Wang ( $\nabla$ enjuwang@163.com )

Hainan Normal University https://orcid.org/0000-0001-5014-6989

\section{Research Article}

Keywords: AIE, TICT, coumarin, fluorescence, solvatochromic property

Posted Date: January 11th, 2022

DOI: https://doi.org/10.21203/rs.3.rs-1230235/v1

License: (c) (i) This work is licensed under a Creative Commons Attribution 4.0 International License. Read Full License

Version of Record: A version of this preprint was published at Journal of Fluorescence on April 20th, 2022. See the published version at https://doi.org/10.1007/s10895-022-02933-2. 


\title{
Substituent Effect on AIE Mechanism of Two Coumarin Derivatives: Uncommon TICT Fluorescence in Aggregation State
}

\author{
Qiao $\mathrm{Li}^{1} \cdot$ Yang Zhao ${ }^{1} \cdot$ Zhigang Niu $^{1} \cdot$ Enju Wang ${ }^{1}$ \\ Received: /Accepted: \\ Enju Wang \\ enjuwang@163.com \\ 1 Key Laboratory of Tropical Medicinal Resource Chemistry of Ministry of Education, Key Laboratory of Tropical \\ Medicinal Plant Chemistry of Hainan Province, College of Chemistry \& Chemical Engineering, Hainan Normal University, \\ Haikou, 571158, China
}

\begin{abstract}
$\begin{array}{llll}\text { Two coumarin } & \text { derivatives, } & \text { 7-diethylamino-3-(4-nitrophenyl)coumarin } & \text { (DNC) and }\end{array}$ 7-hydroxy-3-(4-nitrophenyl)coumarin (HNC), were synthesized via Knoevenagel condensation of salicylaldehyde derivatives with 4-nitrophenylacetonitrile and then cyclization reaction. Both of them were characterized by single-crystal X-ray diffraction. The molecules of DNC are stacked via $\pi-\pi$ interaction, while the hydrogen bond interactions instead of $\pi-\pi$ interaction were observed in the crystal packing of HNC. Both of DNC and HNC showed solvatochromic properties and aggregation-induced emission (AIE) activities, but the AIE characteristics of them were entirely different. HNC exhibited an AIE phenomenon as the result of the restriction of twisted intramolecular charge transfer (TICT), while DNC emited peculiar dual fluorescence which was assigned to the emission based on the inhibition of TICT state formation and the emission from the TICT state respectively.
\end{abstract}

Keywords AIE $\cdot$ TICT $\cdot$ coumarin $\cdot$ fluorescence $\cdot$ solvatochromic property 


\section{Introduction}

Conventional luminophores often show intense fluorescent emission in dilute solutions but meet with emission quenching in molecular aggregation state or in solid state, which is widely known as aggregation-caused quenching (ACQ). In recent decades, a class of new functional materials with aggregation-induced emission (AIE) has emerged as a rising hot topic for their widely applications in organic light-emitting diodes [1, 2], dye-sensitized solar cells [3], bioimaging [4], and cancer theranostics [5]. Exploring the relationships between molecular structures and fluorescence properities are essential for the understanding of the AIE mechanism and designing of the high performance AIE-active materials. The generally accepted explanations for AIE mechanisms are the restriction of intramolecular rotation (RIR), vibrations (RIV) and motions (RIM) [6]. In addition to the mechanisms based on molecular geometry, much effort has also been taken to explain the AIE phenomenon based on the electronic effect, such as the restriction of photoinduced twisted intramolecular charge transfer (TICT) [7]. The AIE-active molecules based on RIR, RIV and RIM commonly exhibit twisted molecular configurations which make its synthesis difficult. The structures of TICT-based AIE-active molecules are relatively simple and, in consequence, they are easily obtained [8-10]. Moreover, the combination of AIE and TICT characteristics may produce unique fluorescence performance [11].

In the course of our continuing efforts in understanding the relationship between structure and photoluminescence [12, 13], two coumarin derivatives, 7-diethylamino-3-(4-nitrophenyl)coumarin (DNC) and 7-hydroxy-3-(4-nitrophenyl)coumarin (HNC), were synthesized (Scheme 1) and their structures were determined by single-crystal X-ray diffraction. Both of DNC and HNC showed typical solvatochromic characterics and AIE activities, but the AIE mechanisms of them were entirely different. HNC exhibited an AIE phenomenon as the result of the restriction of TICT state formation, while DNC in the aggregate state emited peculiar dual fluorescence which were ascribed to the relaxation of the locally excited state and the radiative relaxation of TICT state respectively. The TICT fluorescence in aggregation state is extremely rare because the molecular aggregation is usually unfavourable for the formation of the photoinduced TICT state.

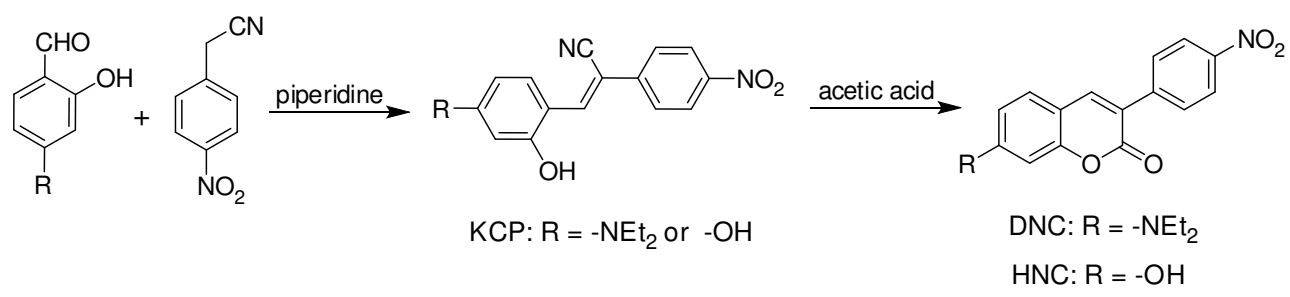

Scheme 1. Synthetic route of target compounds 


\section{Experimental}

\section{Reagents and apparatus}

All chemicals were obtained from commercial suppliers. ${ }^{1} \mathrm{H}$ NMR and ${ }^{13} \mathrm{C}$ NMR spectra were recorded on a Bruker Av400 NMR spectrometer. ESI-MS spectra were performed on a Bruker Esquire HCT mass spectrometer. Fluorescence spectra were taken on a Hitachi F-7000 fluorescence spectrometer. The X-ray diffraction data was collected on an Agilent Gemini A Ultra diffractometer.

\section{General synthetic procedure for DNC and HNC}

Salicylaldehyde derivative (4-diethylaminosalicylaldehyde or 4-hydroxysalicylaldehyde, 4 mmol) and 4-nitrophenylacetonitrile $(0.648 \mathrm{~g}, 4 \mathrm{mmol})$ were dissolved in absolute ethanol $(5 \mathrm{~mL})$. After a tiny amount of piperidine was added for catalysis, the mixture was stirred for $2 \mathrm{~h}$ at $60{ }^{\circ} \mathrm{C}$. A red solid precipitated out. The precipitate was collected by filtration and then washed several times with ethanol to give the intermediate KCP. $\mathrm{KCP}(2 \mathrm{mmol})$ in acetic acid $(5 \mathrm{~mL})$ was stirred for $0.5 \mathrm{~h}$ at $110{ }^{\circ} \mathrm{C}$ during which time a solid precipitated out. The precipitate was collected by filtration and then washed several times with a small amount of ethanol respectively to afford DNC and HNC.

DNC: orange solid, yield: $90.2 \%,{ }^{1} \mathrm{H}$ NMR (400 MHz, DMSO-d6) $\delta: ~ 8.34 ~(s, 1 H), 8.27$ (d, 2H, J = 8.2 Hz), $8.04(\mathrm{~d}, 2 \mathrm{H}, J=8.2 \mathrm{~Hz}), 7.56(\mathrm{~d}, 1 \mathrm{H}, J=8.2 \mathrm{~Hz}), 6.78(\mathrm{~d}, 1 \mathrm{H}, J=8.2 \mathrm{~Hz}), 6.60(\mathrm{~s}, 1 \mathrm{H}), 3.47(\mathrm{q}, 4 \mathrm{H}, J=6.4$ $\mathrm{Hz}), 1.15(\mathrm{t}, 6 \mathrm{H}, J=6.4 \mathrm{~Hz}),{ }^{13} \mathrm{C}$ NMR $\left(100 \mathrm{MHz}, \mathrm{DMSO}-\mathrm{d}_{6}\right) \delta 159.9,156.2,151.2,145.9,143.1,142.4,130.3$, 128.6, 123.2, 115.8, 109.4, 108.2, 96.0, 44.1, 12.2. ESI-MS m/z calculated for $[\mathrm{M}+\mathrm{H}]^{+} 339.13$, found 339.3 .

HNC: yellow solid, yield: 86.5 \%, ${ }^{1} \mathrm{H}$ NMR (400 MHz, DMSO-d6) $\delta: 10.79$ (s, 1H), 8.39 (s, $\left.1 \mathrm{H}\right), 8.29$ (d, $2 \mathrm{H}, \mathrm{J}=8.8 \mathrm{~Hz}), 8.01(\mathrm{~d}, 2 \mathrm{H}, J=8.8 \mathrm{~Hz}), 7.65(\mathrm{~d}, 1 \mathrm{H}, J=8.8 \mathrm{~Hz}), 6.85\left(\mathrm{dd}, 1 \mathrm{H}, J_{1}=8.8 \mathrm{~Hz}, J_{2}=2.0 \mathrm{~Hz}\right), 6.77$ $(\mathrm{d}, 1 \mathrm{H}, J=2.0 \mathrm{~Hz}) .{ }^{13} \mathrm{C}$ NMR $\left(100 \mathrm{MHz}, \mathrm{DMSO}-\mathrm{d}_{6}\right) \delta 162.7,160.1,155.9,147.1,143.7,142.4,131.1,129.8$, 123.8, 120.3, 114.2, 112.2, 102.3. ESI-MS m/z calculated for [M-H] 282.04, found 282.0.

\section{Crystallography}

Single crystals of DNC and HNC suitable for X-ray analysis were obtained by slow evaporation of their THF solutions at room temperature. The diffraction data collection was performed at $293 \mathrm{~K}$ on a Gemini A Ultra diffractometer $(\mathrm{MoK} \alpha, \lambda=0.71073 \AA$ ) $)$. The intensity data were corrected for Lorentz and polarization effects. The structures were solved by direct methods and all non-hydrogen atoms were refined anisotropically by the full-matrix least-squares technique using the SHELXL-2014/7 package [14]. All the hydrogen atoms were set in geometrically calculated positions and refined isotropically. The elementary crystal data were provided in supplementary materials (Table S1). For more information, see crystallographic information files that have 
been deposited in the Cambridge Crystallographic Data Centre (CCDC 2130543 for DNC and 2130544 for HNC).

\section{Results and Discussion}

\section{Crystal Structure}

DNC crystallizes in monoclinic system, space group $P 2_{1} / c$. As shown in Fig. 1A, the 4-nitrophenyl moiety and the coumarin moiety are not coplanar with a dihedral angle of $36.6^{\circ}$. The $\mathrm{N}$ atom of the diethylamino moiety exhibits $\mathrm{sp}^{2}$ hybridization and form a $\mathrm{p}-\pi$ conjugated system with the coumarin moiety. The dihedral angle between the plane through $\mathrm{N}(1), \mathrm{C}(16), \mathrm{C}(18)$ and the coumarin plane is $15.0^{\circ}$. The molecules are stacked along the $b$-axis to form one-dimensional molecular chains (Fig. 1B). The adjacent molecules in the molecular chain interact via $\pi-\pi$ stacking interaction with a distance of $3.6170 \AA$ between the centroid of benzene and the coumarin plane.

HNC crystallizes in triclinic system, space group $P-1$. There are two independent molecules in crystal lattice and they exhibit distinct conformation differences. In one molecule the dihedral angle between the plane through phenyl moiety and the plane through coumarin moiety is $20.1^{\circ}$, while it is $25.1^{\circ}$ in the other molecule (Fig. 2A). The two types of unique molecules stack separately along the $c$-axis to form two molecular chains which are linked by two hydrogen bonds $(\mathrm{O}(42)-\mathrm{H}(42) \cdots \mathrm{O}(21)$ and $\mathrm{O}(20)-\mathrm{H}(20) \cdots \mathrm{O}(41))$ (Table S2). No $\pi-\pi$ stacking interactions were found in the crystal packing (Fig. 2B).

(A)

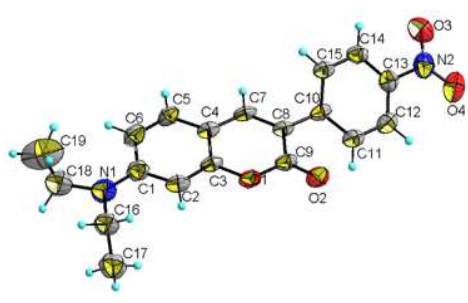

(B)

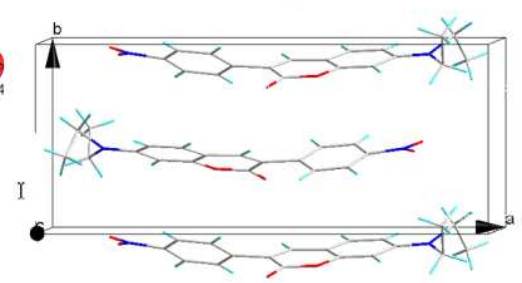

Fig. 1 (A) Crystal structure of DNC shown at 50\% probability. (B) Crystal packing showing one-dimensional molecular chain along $b$-axis via $\pi-\pi$ stacking interaction. 


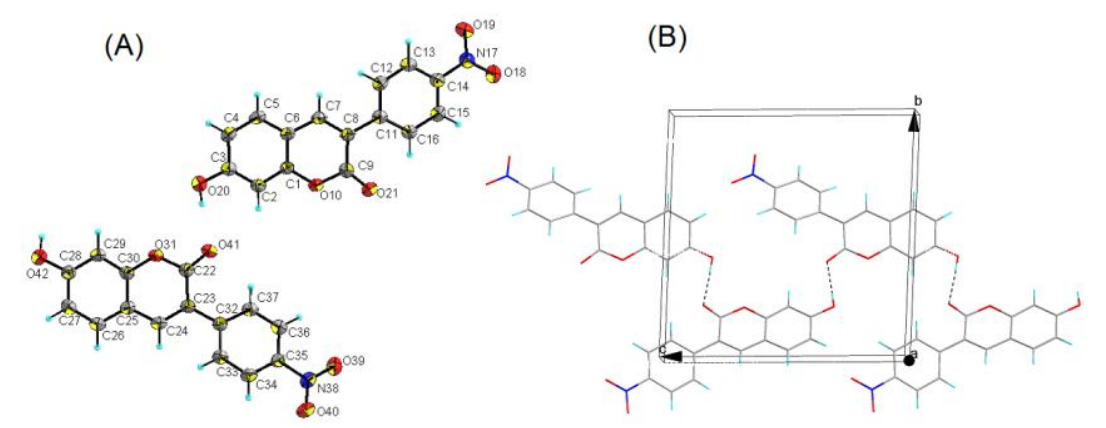

Fig. 2 (A) Crystal structure of HNC shown at 50\% probability. (B) Crystal packing viewed along $a$-axis.

\section{Solvatochromic Properties}

Both of DNC and HNC are the typical donor-acceptor type luminophores which commonly show solvent-dependent photophysical property $[15,16]$. Therefore, the solvent polarity effect on the emission of them was firstly investigated and the fluorescent emission spectra of them $(10 \mu \mathrm{mol} / \mathrm{L})$ in different solvents are shown in Fig. 3. The emission peak of DNC appears at $475 \mathrm{~nm}$ in petroleum ether, which shifted to 505 $\mathrm{nm}$ in toluene due to the solvent-enhanced intramolecular charge transfer (ICT). In more polar solvents such as THF, MeCOOEt and $\mathrm{CHCl}_{3}$ the luminescence intensities of the DNC were dramatically weakened, and even were quenched in $\mathrm{MeCN}, \mathrm{EtOH}$ and $\mathrm{MeOH}$ owning to the conversion of from the locally excited state to the dark twisted intramolecular charge transfer state (Fig. 3A). The emission colors of DNC in different solvents under $365 \mathrm{~nm}$ UV lamp were illustrated in Fig 3B. The blue luminescence in petroleum ether and the strong green luminescence in toluene were observed. The emission of DNC in THF, MeCOOEt and $\mathrm{CHCl}_{3}$ was so faint that it is hard to be distinguished. In $\mathrm{MeCN}$, $\mathrm{EtOH}$ and $\mathrm{MeOH}$, no luminescence was perceived. HNC displayed similar solvatochromic property as DNC. With the increase of the solvent polarity (from petroleum ether to acetonitrile), the emission peak gradually shifted to long-wavelength region and the fluorescence intensity is enhanced except for $\mathrm{MeCN}$ in which the emission intensity decreased. In EtOH and $\mathrm{MeOH}$ the fluorescence emission was neatly quenched (Fig. 3C). The fluorescence color change from blue to orange was observed when the solvent changed from petroleum ether to acetonitrile. In the more polar solvents such as ethanol and methanol, the fluorescence of HNC was quenched due to the formation of dark TICT state (Fig. 3D). 

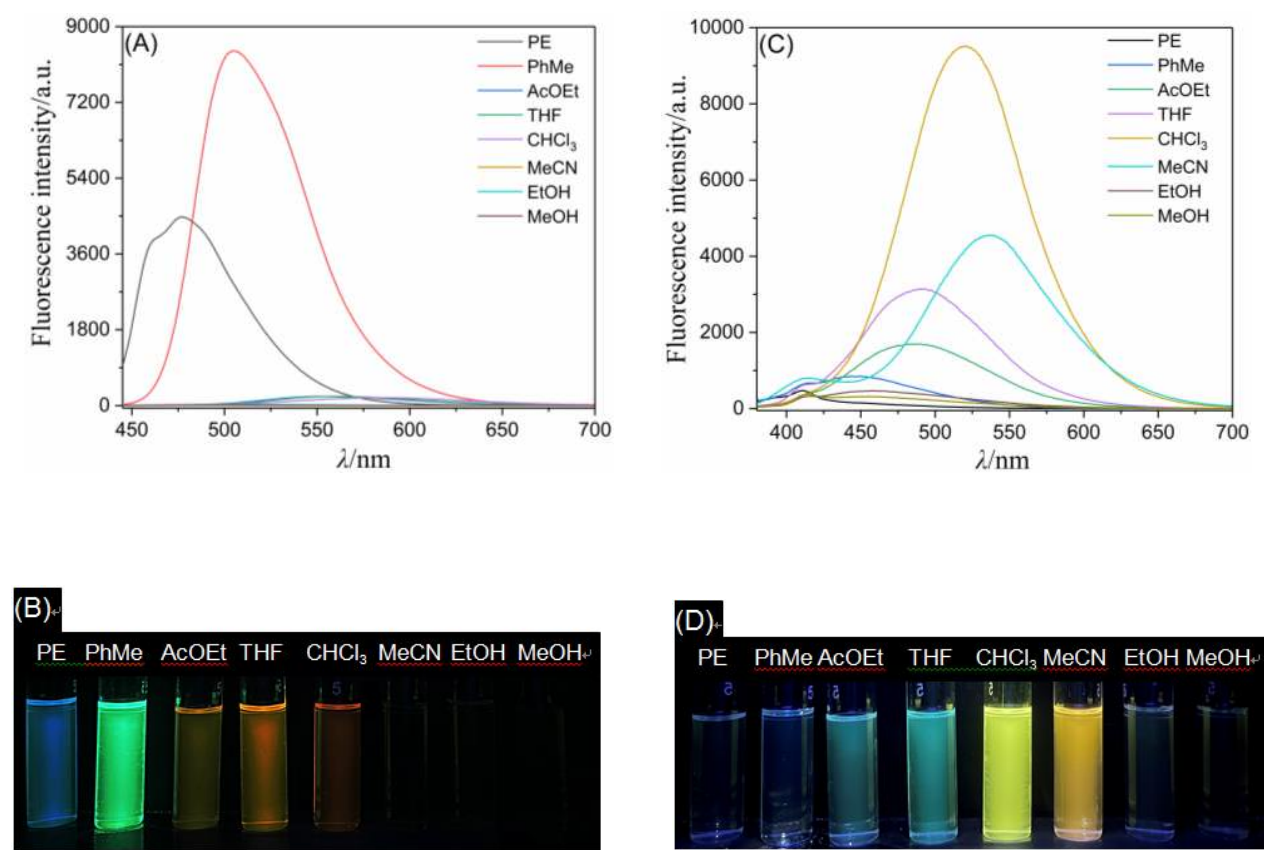

Fig. 3 Fluorescence spectra of DNC (A) and HNC (C) in different solvents. Photographs of DNC (B) and HNC (D) in different solvents taken in a dark-box ultraviolet analyzer when excited at $365 \mathrm{~nm}$.

\section{AIE Properties}

The AIE characteristics of DNC and HNC in the binary mixtures of acetonitrile and water with varied volume ratios were investigated. As illustrated in Fig. 4A-C, HNC emitted strong fluorescence in pure acetonitrile. When increasing the water fraction from 0 to $10 \%$, its emission was dramatically quenched due to the formation of TICT non-emissive state. In $\mathrm{MeCN} / \mathrm{H}_{2} \mathrm{O}$ solutions with water fractions between $10 \%$ and $70 \%$, little changes in emission spectra were observed. When the water fraction was increased from $70 \%$ to $90 \%$, the fluorescence intensity was distinctly enhanced and accompanied by a little blue shift, which was ascribed to the fact that the formation of the molecular aggregates inhibited the formation of TICT state and thereby local fluorescence appered. Unlike HNC exhibiting AIE characteristics based on the TICT mechanism that has been widely reported [17-23], DNC exhibied peculiar dual fluorescence in the aggregate state. As shown in Fig. 4D-F, DNC emitted weak green fluorescence at $485 \mathrm{~nm}$ in pure acetonitrile. Upon adding water, the emission intensities were reduced slightly and about $10 \mathrm{~nm}$ red-shift was observed. When increasing the water fraction from 60 to $99 \%$, a slight emission enhancement at $495 \mathrm{~nm}$ occurred and accompanied by a new emission peak at about $625 \mathrm{~nm}$. The appearance of the dual fluorescence should be ascribed to the formation of molecular aggregates in $\mathrm{MeCN} / \mathrm{H}_{2} \mathrm{O}$ solutions with water fractions up to $60 \%$. The AIE effect of DNC was unconspicuous on account of the $\pi-\pi$ stacking interaction in the aggregates of 
DNC (Fig. 1B). The shorter-wavelength emission $(495 \mathrm{~nm})$ of the dual fluorescence was assigned to the relaxation of the locally excited state, while the longer-wavelength emission $(625 \mathrm{~nm})$ should be ascribed to the radiative relaxation of TICT state. It suggested that the aggregation cannot entirely inhibit the TICT state formation, and the TICT state in the aggregation state tend to relax back to the ground state via radiative channels. The TICT fluorescence in solution is rarely observed because the TICT state often relaxes back to the ground state via non-radiative pathways [15]. The aggregation-state TICT fluorescence is more rare because the molecular aggregation generally cause the restriction of the photoinduced TICT state formation [24]. On the contrary, the aggregation-induced TICT restriction can commonly lead to local fluorescence [16]. Therefore, the aggregation-state TICT fluorescence of DNC is a very interesting finding.

The photos of DNC and HNC in solid states under UV light irradiation and natural light were shown in Fig. 5. DNC was orange under natural light (Fig. 5C). When excited by UV irradiation, it emitted strong red fluorescence both in the crystal state and the powder state (Fig. 5A and 5B), whereas its dual fluorescence in $\mathrm{MeCN} / \mathrm{H}_{2} \mathrm{O}$ mixtures is pink to rose (different mixture of green and red fluorescence, Fig. 4F). It suggested that DNC in solid states is more propitious to emit TICT fluorescence than its aggregates in $\mathrm{MeCN} / \mathrm{H}_{2} \mathrm{O}$ mixtures. In general, organic compounds in the crystal state were unable to emit TICT fluorescence because the tight, regular molecular arrangement in crystal lattices restricts the change in the molecular geometry for the formation of the TICT state. Hence the TICT fluorescence in solid state is extremely rare except for the particular-shaped molecules [25]. HNC in solid states was yellow under natural light (Fig. 5F). Under UV light excitation it emitted green fluorescence both in crystal and powder state as observed in $\mathrm{MeCN} / \mathrm{H}_{2} \mathrm{O}$ mixtures (Fig. 7D and E). 

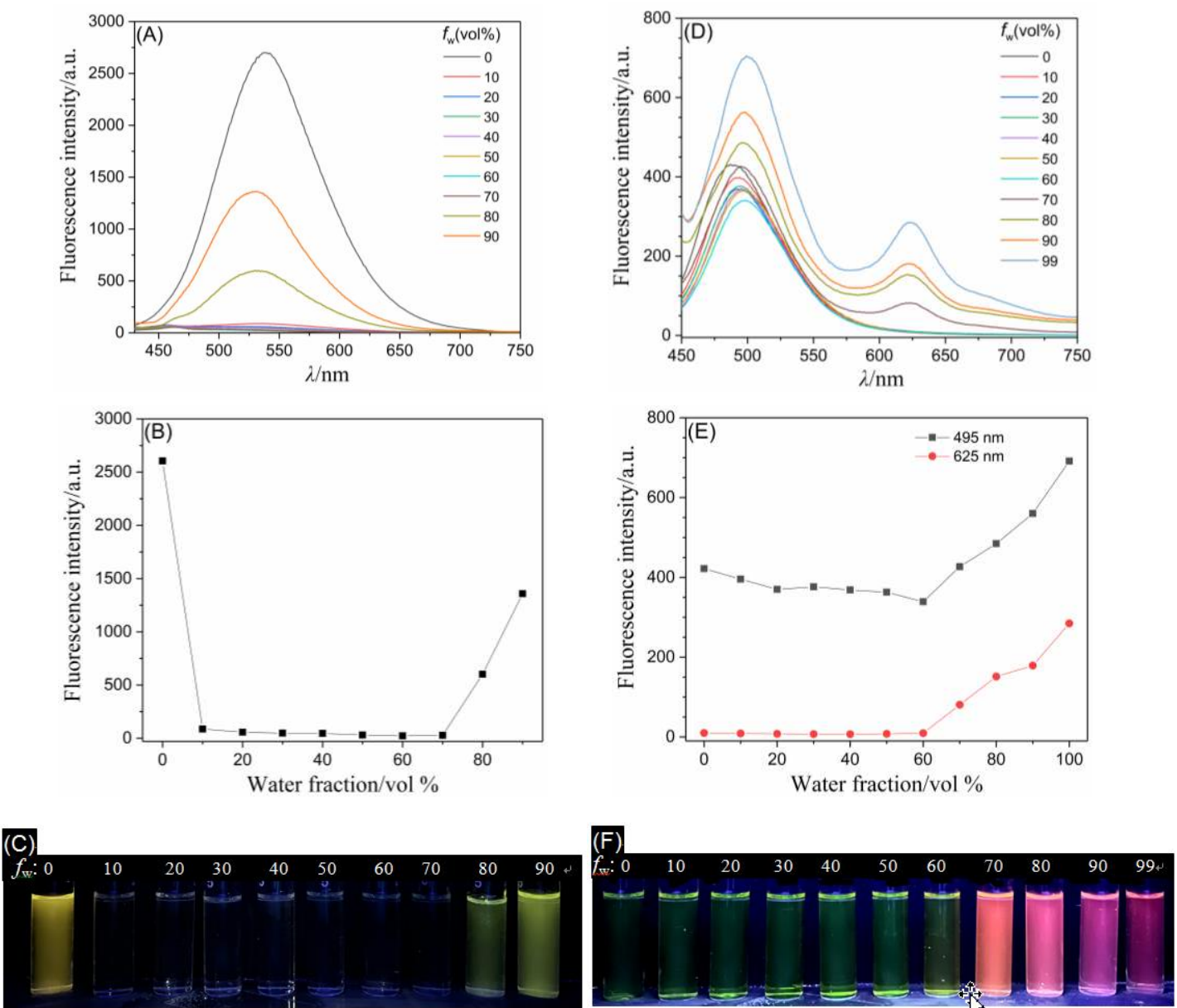

Fig. 4 Fluorescence spectra of $\mathrm{HNC}(\mathrm{A})$ and $\mathrm{DNC}$ (D) in $\mathrm{CH}_{3} \mathrm{CN} / \mathrm{H}_{2} \mathrm{O}$ mixtures with different water fractions. Plots of fluorescence intensity versus water fraction for $\mathrm{HNC}(\mathrm{B})$ and DNC (E). Photographs of $\mathrm{HNC}(\mathrm{C})$ and $\mathrm{DNC}(\mathrm{F})$ in $\mathrm{MeCN} / \mathrm{H}_{2} \mathrm{O}$ with different water fractions under 365 nm UV irradiation. The concentrations of $\mathrm{HNC}$ and $\mathrm{DNC}$ are $50 \mu \mathrm{mol} / \mathrm{L}$ and $10 \mu \mathrm{mol} / \mathrm{L}$ respectively
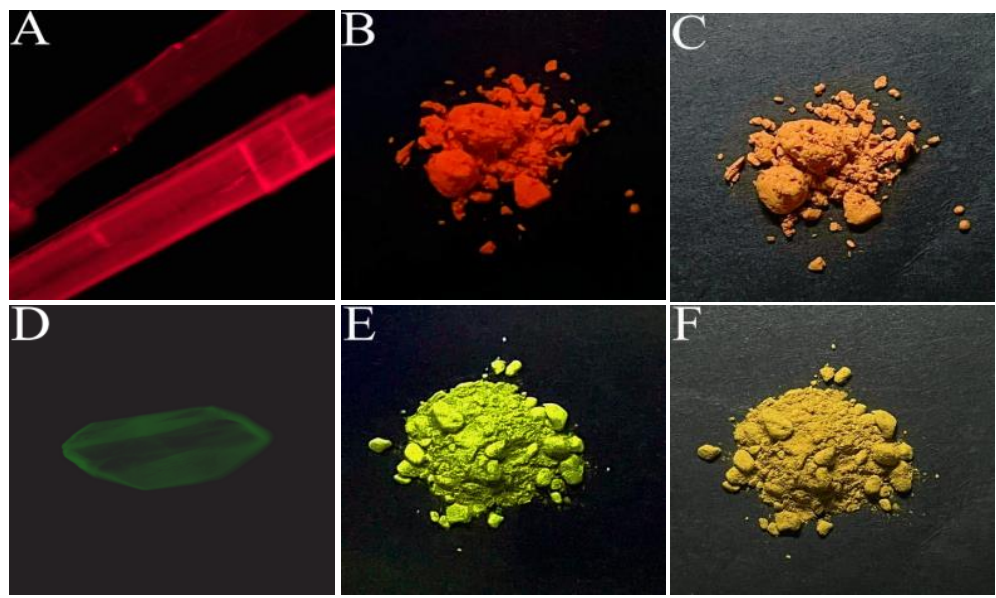

Fig. 5 Fluorescent photos under UV irradiation: (A) DNC in crystal state; (B) DNC in amorphous solid state; (D) HNC in crystal state; (E) HNC in amorphous solid state, and the photos under natural light: (C) DNC; (F) HNC 


\section{Conclusion}

In summary, we have obtained two coumarin-based donor-acceptor type luminophores which were briefly named as DNC and HNC through a facial synthetic strategy. HNC exhibited typical AIE characteristics based on the mechanism of the restriction of TICT state formation in the aggregate state. Nevertheless, DNC in aggregate state emited dual fluorescence which were ascribed to the emission based on the restriction of TICT state formation and the TICT emission respectively. The TICT emission in the aggregate state is very rare.

Supplementary Information The online version contains supplementary material available at https://

\section{Declarations}

Funding This work is financially supported by the National Natural Science Foundation of China (No. 22061016) and Program for Innovative Research Team in University (IRT-16R19).

Conflicts of Interest/Competing Interests The authors have no conflicts of interest to declare that are relevant to the content of this article.

Ethical Approval Not applicable.

Consent to participate Not applicable.

Consent for publication Not applicable.

Availability of data and material/ Data availability The crystallographic information files that have been deposited in the Cambridge Crystallographic Data Centre (CCDC 2130543 for DNC and 2130544 for HNC) which are freely available for all.

The link for CCDC: https://www.ccdc.cam.ac.uk/.

Code Availability Not applicable.

Author Contributions EW contributed to the study conception and design, and was the major contributor in writing the manuscript. The synthesis, spectrum test, data analysis and so on were performed by QL with the assistance of YZ. The X-ray diffraction data collection and structure determination was performed by $\mathrm{ZN}$. 


\section{References}

1. Cai Y, Qin A, Tang BZ (2017) Siloles in optoelectronic devices. J Mater Chem C 5: 7375-7389. https://doi.org/10.1039/C7TC02511D

2. Liu D, Wei JY, Tian WW, Jiang W, Sun YM, Zhao Z, Tang BZ (2020) Endowing TADF luminophors with AIE properties through adjusting flexible dendrons for highly efficient solution-processed nondoped OLEDs. Chem Sci 11: 7194-7203. https://doi.org/10.1039/D0SC02194F

3. Li CT, Kuo YL, Kumar CHP, Huang PT, Lin JT (2019) Tetraphenylethylene tethered phenothiazine-based double-anchored sensitizers for high performance dye-sensitized solar cells. J Mater Chem A 7: 23225-23233. https://doi.org/10.1039/C9TA09025H

4. Qian J, Tang BZ (2020) AIE luminogens for bioimaging and theranostics: from organelles to animals. Chem 3: 56-91. http://dx.doi.org/10.1016/j.chempr.2017.05.010

5. Gao M, Tang BZ (2020) AIE-based cancer theranostics. Coordin Chem Rev 402: 213076. https://doi.org/10.1016/j.ccr.2019.213076

6. Mei J, Leung NLC, Kwok RTK, Lam JWY, Tang BZ (2015) Aggregation-induced emission: together we shine, united we soar. Chem Rev 115: 11718-11940. https://doi.org/10.1021/acs.chemrev.5b00263

7. Gao BR, Wang HY, Hao YW. Fu LM, Fang HH, Jiang Y, Wang L, Chen QD, Xia H, Pan LY, Ma YG., Sun HB (2010) Time-resolved fluorescence study of aggregation-induced emission enhancement by restriction of intramolecular charge transfer state. $J$ Phys Chem B 114: 128-134. https://doi.org/10.1021/jp909063d

8. Abedi SAA, Chi W, Tan D, Shen T, Wang C, Ang ECX, Tan CH, Anariba F, Liu X (2021) Restriction of twisted intramolecular charge transfer enables the aggregation-induced emission of 1-(N,N-dialkylamino)-naphthalene derivatives. J Phys Chem A 125: 8397-8403. https://doi.org/10.1021/acs.jpca.1c06263

9. Qian Y, Cai MM, Xie LH, Yang GQ, Wu SK, Huang W (2011) Restriction of photoinduced twisted intramolecular charge transfer. ChemPhysChem 12: 397-404. https://doi.org/10.1002/cphc.201000457

10. Li J, Qian Y, Xie L, Yi Y, Li W, Huang W (2015) From dark TICT state to emissive quasi-TICT state: the AIE mechanism of $\quad \mathrm{N}$-(3-(benzo[d]oxazol-2-yl)phenyl)-4-tert-butylbenzamide. J $\quad$ Phys $\quad$ Chem $\quad \mathrm{C} 119$ : $2133-2141$. https://doi.org/10.1021/jp5089433

11. Chiang Y, Lai Z, Chen C, Chang C, Liu B (2018) Construction of emission-tunable nanoparticles based on a TICT-AIEgen: Impact of aggregation-induced emission versus twisted intramolecular charge transfer. J Mater Chem B 6: 2869-2876. https://doi.org/10.1039/C8TB00539G

12. Li Q, Niu ZG, Liu YL, Wang EJ (2020) Crystal structure and aggregation-induced emission of an azine derivative. Chin J Struct Chem 39: 693-697. https://doi. 10.14102/j.cnki.0254-5861.2011-2464

13. Zhang ZY, Niu ZG, Wang EJ (2019) Chemical conversion of a coumarin derivative containing arylhydrazono: crystal structures and fluorescence properties. Chin J Struct Chem 38: 384-389. https://doi.10.14102/j.cnki.0254-5861.2011-2098

14. Sheldrick GM (2014) SHELXL-2014/7. Program for the Solution of Crystal Structures. University of Göttingen, Göttingen

15. Sasaki, S.; Drummen, G. P. C.; Konishi, G. Recent advances in twisted intramolecular charge transfer (TICT) fluorescence and related phenomena in materials chemistry. J. Mater. Chem. C 2016, 4, 2731-2743.

https://doi.org/10.1039/C5TC03933A 
16. Sun H, Tang XX, Miao BX, Yang Y, Ni Z (2018) A new AIE and TICT-active tetraphenylethene-based thiazole compound: synthesis, structure, photophysical properties and application for water detection in organic solvents. Sensor Actuat B-Chem 267: 448-456. https://doi.org/10.1016/j.snb.2018.04.022

17. Wang DH, Chen LJ, Zhao X, Yan XP (2021) Enhancing near-infrared AIE of photosensitizer with twisted intramolecular charge transfer characteristics via rotor effect for AIE imaging-guided photodynamic ablation of cancer cells. Talanta 225: 122046. https://doi.org/10.1016/j.talanta.2020.122046

18. Hu R, Lager E, Aguilar-Aguilar A, Liu J, Lam JWY, Sung HHY, Williams ID, Zhong Y, Wong KS, Peña-Cabrera E, Tang BZ (2009) Twisted Intramolecular Charge Transfer and Aggregation-Induced Emission of BODIPY Derivatives. J Phys Chem C 113: 15845-15853. https://doi.org/10.1021/jp902962h

19. Zhang J, Xu B, Chen J, Wang L, Tian W (2013) Oligo(phenothiazine)s: twisted intramolecular charge transfer and aggregation-induced emission. J Phys Chem C 117: 23117-23125. https://doi.org/10.1021/jp405664m

20. Sun H, Tang XX, Miao BX, Yang Y, Ni Z (2018) A new AIE and TICT-active tetraphenylethene-based thiazole compound: synthesis, structure, photophysical properties and application for water detection in organic solvents. Sensor Actuat B-Chem 267: 448-456. https://doi.org/10.1016/j.snb.2018.04.022

21. Sun H, Tang XX, Zhang R, Sun WH, Miao BX, Zhao Y, Ni ZH (2020) Tetraphenylethene-substituted benzothiadiazoles: AIE and TICT properties, tunable intramolecular conjugation and application in detecting trace water in organic solvents. Dyes Pigments 174: 108051. https://doi.org/10.1016/j.snb.2018.04.022

22. Hu Q, Gong T, Mao Y, Yin Q, Wang Y, Wang H (2021) Two-phase activated colorimetric and ratiometric fluorescent sensor for visual detection of phosgene via AIE coupled TICT processes. Spectrochim Acta A 253: 119589. https://doi.org/10.1016/j.saa.2021.119589.

23. Jiang M, Gu X, Lam JWY, Zhang Y, Kwok RTK, Wong KS, Tang BZ (2017) Two-photon AIE bio-probe with large stokes shift for specific imaging of lipid droplets. Chem Sci 8: 5440-5446. https://doi.org/10.1039/C7SC01400G

24. Zhao Y, He S, Yang J, Sun H, Shen X, Han X, Ni Z (2018) Study on TICT emission of TPE-BODIPY derivatives mediated by methyl group on BODIPY. Opt Mater 81: 102-108. https://doi.org/10.1016/j.optmat.2018.05.023

25. Naito H, Nishino K, Morisaki Y, Tanaka K, Chujo Y (2016) Solid-state emission of the anthracene-o-carborane dyad from the ttwisted-intramolecular charge transfer in the crystalline state. Angew Chem Int Ed 55: 1-7. https://doi.org/10.1002/anie.201609656 


\section{Supplementary Files}

This is a list of supplementary files associated with this preprint. Click to download.

- SupplementaryMaterial.pdf 